\title{
Iranian Shiite Muslim Near-Death Experiences: Features and Aftereffects Including Dispositional Gratitude
}

\author{
Alinaghi Ghasemiannejad, M.A. \\ Shahid Chamran University of Ahvaz \\ Jeffrey Long, M.D. \\ Houma, LA \\ Farnoosh Faith Nouri, Ph.D., LPC-S \\ Dallas, $T X$ \\ Komeyl Farahnakian, M.A. \\ Institute for Humanities and Cultural Studies
}

\begin{abstract}
During nearly four decades of research on near-death experiences (NDEs), Iranian Shiite NDEs have been infrequently reported. Though an early researcher concluded that Muslim NDEs appeared to be rare, later authors concluded they may be common and that their key features may not be very different from those of Western NDEs. The purpose of this study was to further explore NDEs among Iranians, specifically Shiite Muslims. In this study, 33 participants who had survived a close brush with death completed online a demographic questionnaire, a Persian translation of the NDE Scale (Greyson, 1983), and the Persian version of the Gratitude Questionnaire-Six Item Form GQ-6 (McCullough, Emmons, \& Tsang, 2002). Of these participants, 20 (61\%) scored 7 or higher on the Persian NDE Scale, indicating the presence of an NDE. Eight prominent features of Western NDEs were present in our participants' NDEs, and, like Western NDErs, our participants often reported profoundly positive changes in attitudes, values, and spiritual beliefs following their NDEs. Neardeath experiencers indicated significantly more gratitude in life than did survivors of a close brush with death without an NDE, with a medium effect, $t(31)=$ $3.00, p=.005, d=1.04$. Our results showed that NDEs are not rare among Shiite Muslims and may not be particularly different in their main elements from typical Western NDEs. This investigation of NDEs from a non-Western country provides important evidence in the materialist vs. non-materialist debate about the origins of NDEs.
\end{abstract}

KEY WORDS: near-death experience, gratitude, close brush with death, nonWestern near-death experience, Shiite Muslim 
Non-Western near-death experiences (NDEs) may be defined as those reported by people from countries that are not of predominantly Jewish or Christian heritage (Holden, Long, \& MacLurg, 2009). In the first 30 years of NDE research, researchers published at least 65 studies in which they investigated nearly 3,500 near-death experiencers (NDErs), predominantly from Western countries (Holden, Greyson, \& James, 2009). In contrast, during that same time period, investigations on approximately 275-300 non-Western NDEs were published (Kellehear, 2009). A recent analysis of the published scholarly periodical literature on NDEs indicates that a lack of attention to Muslim NDEs persists (Loseu, Holden, Kinsey, \& Christian, 2013). Thus, there is an ongoing need for investigators to conduct studies of nonWestern NDEs with methodology that, to the greatest extent possible, addresses and overcomes the methodological issues inherent in studying them, such as translation between participants' native languages and English.

In 2009, Joel Kreps concluded that Muslim NDEs "appear to be quite rare in fact" (p. 84). Shortly thereafter, two separate investigation teams responded with reports on Muslim NDEs. In one article, Michael Nahm and Joachim Nicolay (2010) reported on eight Muslim NDEs and concluded that "although the documentation standard of the available cases is generally low, these accounts indicate that structure and contents of NDEs from many non-Western Muslim communities are largely similar to those reported in the Western NDE literature" (p. 255). In the second article, Cheryl Fracasso, Seyed Aleyasin, Harris Friedman, and M. Scott Young (2010) reported the following from a survey of Muslim NDErs in Arak City, Iran:

\footnotetext{
Alinaghi Ghasemiannejad, M.A., is a doctoral student in the Department of Psychology at Shahid Chamran University of Ahvaz in Ahvaz, Iran. His primary area of scholarly inquiry is research on the nature, content, and aftereffects of near-death experiences. Jeffrey Long, M.D., is founder of the Near Death Experience Research Foundation (nderf.org). His primary area of scholarly inquiry is evidence regarding the reality and spiritual content of near-death experiences. Farnoosh Faith Nouri, Ph.D., LPC-S, is an educator, Licensed Professional Counselor, Clinical Hypnotherapist, and Board Certified Coach in private practice at LifePulse Center in Dallas, TX. She has been a mental health practitioner since 1997 and higher education teacher since 1993. Presently, she is an adjunct faculty member at Texas Woman's University in Denton, TX. Komeyl Farahnakian, M.A., is a student of the philosophy of religion at the Institute for Humanities and Cultural Studies in Qom, Iran. His primary area of scholarly inquiry is validation of near-death experiences in Islamic theology. The authors would like to thank Ms. Somayeh Nabian and Mr. Amir Ghaneie for their help in translating Persian NDEs into English. We are also grateful to Dr. Janice Holden for her support and encouragement with this project. Correspondence regarding this article should be sent to Mr. Ghasemiannejad at e-mail: ali.ghasemian@ymail.com.
} 
Our results suggest that Muslim NDEs may actually be quite common, as they are in the West, and may not be especially different in their key features from Western NDEs and therefore not heavily influenced by cultural variations, including prior religious or spiritual beliefs. (p. 271)

We saw the potential to make two unique contributions to the neardeath literature. As the majority of people in Iran are Shiite Muslims and no previous researcher had focused on this population, we thought it potentially useful to focus specifically on them. Also, in our review of NDE literature, we detected an aftereffect on which we could not find published research. NDErs often seemed to express an enhanced sense of gratitude for and appreciation of life, including physical existence itself, events, and people (Noyes, Fenwick, Holden, \& Christian, 2009). We were curious how people who survived a close brush with death might respond to a formal assessment of disposition toward gratitude.

In light of the above considerations, we undertook a study of Iranian Shiite Muslim NDEs to (a) examine the content of NDEs among Iranian Shiite Muslims, (b) investigate the similarities and differences between Iranian and Western NDEs and aftereffects, and (c) compare gratitude as an aftereffect between Iranian NDErs and those who survived close brushes with death without NDEs (CBrs).

\section{Method}

\section{Procedures}

We established a website (www.irande.org) in the Persian language to educate Iranians about NDEs and to recruit participants for our study. Visitors to the site saw an announcement about the research, including an invitation for them to participate if they had survived a close brush with death.

The questionnaire was posted on the website. Some participants downloaded the questionnaire, completed it electronically, and returned it as an email attachment. Others printed the form, completed it in hard copy, and returned it by postal mail.

\section{Instruments}

The questionnaire consisted of three instruments-a demographic survey we devised, the NDE Scale (Greyson, 1983) translated into Persian, and the Gratitude Questionnaire GQ-6 (McCullough, Em- 
mons, \& Tsang, 2002) in its Persian form (Ghamarani, Kajbaf, Oreyzi, \& Amiri, 2008) - and a request for respondents to provide a narrative of the circumstances, contents, and aftereffects of their NDEs. Our demographic survey included items regarding age, gender, educational level, religion, length of time since the close brush with death, and circumstances around the experience such as type of accident, illness, or injury.

The NDE Scale (Greyson, 1983) consists of 16 items-four each that assess cognitive, affective, paranormal, and transcendent NDE features. For each item, respondents indicate absence (0), equivocal presence (1), or definite presence (2) of the feature in their experiences during their close brushes with death. Out of a possible maximum score of 32, a score of at least 7 is considered to indicate an NDE. The NDE Scale has shown high rates of reliability and validity in differentiating NDErs from non-NDErs among survivors of a close brush with death (Greyson, 1983, 1997, 2001, 2003, 2007).

Prior investigators have described development of a Persian NDE Scale (Fracasso et al., 2010). Not having access to that version, we developed a Persian version of the NDE Scale for this study. First, coauthor Ghasemiannejad translated the original English NDE Scale (1983) items into Persian, and then two professors at Isfahan University in Isfahan City, Iran, reviewed and modified the translated version. The modified version was then sent for further review to coauthor Nouri, an Iranian American NDE researcher, practitioner, and adjunct professor in Dallas, Texas, bilingual in Persian and English, whose $\mathrm{PhD}$ dissertation addressed electromagnetic aftereffects of NDEs (Nouri \& Holden, 2008). Her further modifications were incorporated, yielding the final version of the Persian NDE Scale used for this study.

Regarding reliability of the NDE Scale, Greyson (1983) found Cronbach's alphas of .88 for the entire Scale and .75 for the cognitive, .86 for the affective, .66 for the paranormal, and .76 for the transcendental components, thus ranging from good to questionable. The Persian NDE Scale yielded a good alpha of .83 for the entire Scale and questionable alphas of .58 for the cognitive, .64 for the affective, .67 for the paranormal, and .68 for the transcendental components. Based on these results, we used only total Scale scores in our analyses.

The GQ-6 (McCullough et al., 2002) is a short, self-report measure of the tendency to experience gratitude in life. Respondents answer 6 items on a 1 to 7 scale ( $1=$ strongly disagree, $7=$ strongly agree); four of the items are positively worded, and two are negatively worded and 
reverse-scored. The GQ-6 has good internal reliability, with alphas between .82 and .87 (McCullough et al., 2002). Results of research in Iran involving a Persian translation of the GQ-6 with a 1 to 5 scale yielded satisfactory convergent validity, internal consistency, testretest, and split-half reliability (Ghamarani et al., 2008). We used this latter version in our questionnaire, and reliability in our sample was an acceptable Cronbach's alpha of .81.

\section{Participants and Analysis}

Participants in this study were a convenience sample of self-identified Shiite Muslims aged 18 years and older and living in various cities in Iran, including Isfahan, Karaj, and Mashhad. Some participants stumbled across the website while searching online, and coauthor Ghasemiannejad came to know others through public speeches and personal meetings and then referred them to the website. Participants submitted completed questionnaires over a 6-month period in 2013.

Regarding quantitative analysis, for participants who scored 7 or higher on the Persian NDE Scale, we calculated mean scores for the total Scale. Using Persian NDE Scale results, we also calculated the percentages of NDErs who endorsed eight selected NDE features that figure prominently in Western NDEs (Zingrone \& Alvarado, 2009). These features include out-of-body experience (OBE), seeing an unearthly light, and life review. For all analyses of differences between NDErs and CBrs, we set alpha level at $p<.05$. In the absence of norms for effect size in NDE research, we used Jacob Cohen's (1988) cautious specifications to assess strength of effects. Regarding qualitative analysis, we noted our informal observations of NDE aftereffect themes (Noyes et al., 2009) in our participants' NDE narratives.

\section{Results}

\section{Quantitative Analysis}

Of 46 submitted questionnaires, 33 were complete and submitted by qualified participants-self-reportedly at least 18 year old, having survived a close brush with death, and being Shiite Muslim. Of these, $20(60.6 \%)$ participants scored 7 or higher on the Persian NDE Scale, thus comprising the NDErs group, and 13 (39.4\%) scored below 7, thus comprising the CBrs group.

Participants' demographics are presented in Table 1. Time since close brush with death for NDErs ranged $0-27$ years $(M=9.00, S D=$ 
Table 1 Participant Demographics

\begin{tabular}{|c|c|c|c|c|}
\hline \multirow[b]{2}{*}{ Demographics } & \multicolumn{2}{|c|}{ NDErs $(n=20)$} & \multicolumn{2}{|c|}{$\operatorname{CBrs}(n=13)$} \\
\hline & $n$ & $\%$ & $n$ & $\%$ \\
\hline \multicolumn{5}{|l|}{ Gender } \\
\hline Men & 14 & 70.0 & 9 & 69.2 \\
\hline Women & 6 & 30.0 & 4 & 30.8 \\
\hline \multicolumn{5}{|l|}{ Education Level } \\
\hline No high school diploma & 2 & 10.0 & 1 & 7.7 \\
\hline High school diploma & 10 & 50.0 & 6 & 45.2 \\
\hline Bachelor's degree & 5 & 25.0 & 4 & 30.8 \\
\hline Master's degree & 1 & 5.0 & 2 & 15.4 \\
\hline Doctoral degree & 2 & 10.0 & 0 & 0.0 \\
\hline \multicolumn{5}{|l|}{ Age } \\
\hline $18-30$ & 5 & 25.0 & 3 & 23.1 \\
\hline $31-50$ & 14 & 70.0 & 8 & 61.6 \\
\hline $51+$ & 1 & 5.0 & 2 & 15.4 \\
\hline \multicolumn{5}{|l|}{ Circumstances of close brush } \\
\hline Serious illness & 2 & 10.0 & 1 & 7.7 \\
\hline Accident-related injury & 8 & 40.0 & 8 & 61.7 \\
\hline Childbirth & 1 & 5.0 & 0 & 0.0 \\
\hline Cardiac arrest & 6 & 30.0 & 2 & 15.4 \\
\hline Suicide attempt & 2 & 10.0 & 0 & 0.0 \\
\hline Not specified & 1 & 5.0 & 2 & 15.4 \\
\hline
\end{tabular}

7.44) and for CBrs ranged 2-26 years $(M=11.38, S D=9.68)$. We calculated chi-square to determine possible differences between groups and found no significant differences with regard to gender, $\mathrm{X}^{2}(1, N=33)$ $=.01, p=.96$; education level, $\mathrm{X}^{2}(4, N=33)=2.40, p=.66$; age, $\mathrm{X}^{2}(2$, $N=33)=1.03, p=.59$; and time since close brush with death, $\mathrm{X}^{2}(20$, $N=33)=20.43, p=.43$.

Regarding Persian NDE Scale scores, the 13 CBrs' total scores ranged 0-6, $M=3.84, S D=2.37$, whereas the 20 NDErs' scores ranged $7-24, M=13.50, S D=5.41$. From the Persian NDE Scale results, we tallied incidence of selected NDE features among the 20 study participants in the NDEr group, both total and subgroup, grouped by gender; results appear in Table 2.

We used an independent groups $t$ test to investigate possible differences in GQ-6 scores between NDErs and CBrs. Results indicated a significant difference with a medium effect, $t(31)=3.00, p=.005$, $d=1.06$. On average, the 20 NDErs indicated significantly more gratitude (range $=20-30, M=26.35, S D=3.34$ ) than did the $13 \mathrm{CBrs}$ (range $=14-30, M=22.00, S D=5.00$ ). 
Table 2 Percentage of Iranian NDErs Reporting Eight Selected NDE Features Assessed by the NDE Scale

\begin{tabular}{|c|c|c|c|c|c|c|}
\hline \multirow[t]{2}{*}{ Feature } & \multicolumn{2}{|c|}{$\operatorname{Men}(n=14)$} & \multicolumn{2}{|c|}{ Women $(n=6)$} & \multicolumn{2}{|c|}{ All $(N=20)$} \\
\hline & $n$ & $\%$ & $n$ & $\%$ & $N$ & $\%$ \\
\hline OBE & 8 & 57.1 & 4 & 66.7 & 12 & 60.0 \\
\hline $\begin{array}{l}\text { Passing into or through a } \\
\text { tunnel }\end{array}$ & 1 & 7.1 & 1 & 16.7 & 2 & 10.0 \\
\hline Positive emotions or feelings & 8 & 57.1 & 4 & 66.7 & 12 & 60.0 \\
\hline $\begin{array}{l}\text { Encountering an unearthly } \\
\text { light }\end{array}$ & 6 & 42.8 & 4 & 66.7 & 10 & 50.0 \\
\hline $\begin{array}{l}\text { Encountering other beings } \\
\text { (mystical beings or deceased } \\
\text { relatives or friends) }\end{array}$ & 5 & 35.7 & 3 & 50.0 & 8 & 40.0 \\
\hline $\begin{array}{l}\text { A sense of alteration of time } \\
\text { and place }\end{array}$ & 14 & 100.0 & 3 & 50.0 & 17 & 85.0 \\
\hline Life review & 6 & 42.8 & 2 & 33.3 & 8 & 40.0 \\
\hline $\begin{array}{l}\text { Encountering unworldly } \\
\text { realms }\end{array}$ & 4 & 28.6 & 3 & 50.0 & 7 & 35.0 \\
\hline
\end{tabular}

\section{Qualitative Data}

To give readers a feel for the qualitative nature of Shiite Muslims' NDEs as well as a sense of NDE aftereffects, we present three NDE narratives translated from Persian to English. We selected these three because they seemed representative of the narratives our 20 NDEr participants provided.

\section{Mr. Abolhamid's Narrative}

After a military operation when Iran reclaimed the city of Paveh from Iraq, there was another small operation in the west side, in which a bullet hit me in the head and I went into coma. I was assumed dead because my pulse was extremely weak and could not be detected. They wrapped my body in a plastic bag and moved me back with all the other corpses and put me into a cold storage. However, they finally noticed vapor accumulating inside the plastic bag my body was in, and realized that I am alive. I was in coma for about a month. The only memory I have from my coma was that I was walking on a path and could hear people talking, that is all. When I woke up from my coma I had lost my memory. I could not even read. Afterwards they took me to a surgery. During the surgery, although I was anesthetized, I 
could hear the doctors and nurses talking. I could even see what color of dress they were wearing. I was watching the whole thing from the ceiling.

I should say that I had these kinds of experiences [previously]. The first one was in a surgery in 1986 during the Iran-Iraq war, when I was under anesthesia. I was feeling that I am carried along a way on a rail or roller or something. After I woke up, I could not remember who I was or what my name or address was, and I didn't have my military tag on me either. The second time was in Loghman-Hakim hospital in Tehran where I had a few surgeries and I could hear the staff talking while I was under operation and could see the color of their outfit. I could hear their jokes. Another time was in 1990 or 1991. I was in a very bad health condition due to my injuries in the war and had a seizure. My mother told me later that I went through cardiac arrest and she had cried and prayed a lot, and I think that is what pulled me back.

There was no fear during any of these experiences; on the contrary, there was a special feeling of peace and warmth. I could feel the difference between the physical and spiritual world. I could feel that physical laws, like gravity or pain, do not apply to this spirit world. You can see clearly in the dark of the night and you can look right into the sun without burning your eyes. I could feel being protected by an invisible force. I didn't feel lonely or lost at all, it is hard to describe. It felt like there were eyes watching me but I could not see them. Although one expects to feel distressed, the dominant feeling was love. But I did miss my loved ones. I was surrounded by light with a special color of green and yellow. I felt being on some kind of edge or boundary, where one side had one color and the other side a different color. I could go to one side and return. I could see the light and colors, but could not see any source for it.

Being right on the boundary of physical and metaphysical worlds was a pleasant experience. You want to repeat that feeling and always chase it. This brings you close to an existential experience. This experience gives you a feeling that there is something beyond that boundary. When you get away from that green atmosphere, it is very unpleasant to get dirty again and come back to the physical world.

In my experience, when I was in the hospital, there was a nurse whose dress was not proper and was exposed. But during my experience, I saw that she was shining with light. I was wondering who she is, but then saw her feeding me personally by putting food in my mouth. She was very caring about a wounded soldier who is weak and handicapped and lonely and has no one else there. She was doing it with so much compassion. So you see that there is something going on. What they (prophets and spiritual leaders) say is not just fairy tales. This view helps you from sinking in this physical world, of course if you don't deny the spiritual aspect of the experience.

You don't feel lonely there; it is like eyes are watching you. We in- 
terpret things according to our own religious teachings. We might call them angels or something, but this is our interpretation. You know there is something but you don't know how it looks like and cannot paint a picture. I also saw an image of myself when I was very old, and I looked soaked. Like Ammar in the TV series Imam Ali.

\section{Mr. Behnam's Narrative}

At that time, my life was disintegrated and I was living a nervous life. God has no place in that life and I felt that I am rejected. I was living alone, and my life was a real mess and I didn't know what to do. My wife had been cheating on me, and I was wondering what wrong deeds I had done to her; I had supported her financially and all her needs were met during our marriage. I took a firm stand to commit suicide and cut my vein five times. Blood was oozing all around me. I remember nothing until I opened my eyes in Khorshid Hospital. The ambulance took me to the Gharazi Hospital at first, and they rejected the body and announced me dead. Nevertheless, Khorshid Hospital had accepted me though the medical staff was amazed by my revival.

I was absolutely sure of my death; and the question was who took me to the hospital because I surely knew that nobody was home when I committed suicide. After a while I remembered some scene of that incident; I remembered that I separated from my body and exposed to a tunnel of light. I didn't want to go to the tunnel willingly, it vacuumed me. There was no way out, just a light that I was on the path to reach it. This happened instantly as if there was no distance between.

Then I heard some vague voices. I was truly delighted, and I knew this place is not a dream and I am not hallucinating. I happily said "I arrive!" then I felt two presences. No word can explain them, they didn't have any body or any shape. They were very angry and furious. Without expressing any word they sent this feeling to me that I was not allowed there! Then they returned me back. In my way back I felt such an extreme torment that accompanies me from that time on. Then I opened my eyes in hospital and my sister was the first person whom I saw. I asked her what was happening and why I was there?

After that my mind was full of unanswered questions about this event; why I came back? Why they forced me to come back? Why I am not allowed to commit suicide? All my knowledge of death and dying was changed after my return. I know very well that happening revived me and gave me a new impression about life. Now I truly believe that death is the beginning of a new story. Based on a verse Quran "we are from God and will come back to Him." Every Muslim knows these words, and whenever one passes away we hear this sentence. So think deeply about it again; Our uniting with God is guaranteed through death. The hardship of life keeps us away from God, so while we are living we can't fully sense Him. 
Now I am not afraid of death any more. And try my best to live a complete life so that I shall readily accept death any time. I eagerly want to meet my death; furthermore, I don't ruin the opportunity of living by waiting for death.

As an ending point I would like to say that we are fascinated by the materials and the beauty of the world that we forgot the presence of God. These beauties limit us to this world and don't let us think of the Creator.

\section{Mrs. Batool's Narrative}

This story happened four years ago when I was in a hospital for natural [childbirth] delivery, and it was after the delivery that I wanted to use the bathroom with the help of my sister. As I was leaving the bathroom, suddenly all things around me went into gray and I felt as if I am falling down with high speed.

I saw gray walls with recognizable bricks. I saw people that were working on this wall; abruptly, I changed the direction upward with a loud noise and higher speed. I was going to a gate from which a light was coming. As I came closer to the light it became shinier, more beautiful and more gorgeous, even I could see colorful particles of the light. Furthermore, I felt a delighted and pleased breeze, and I breathed it in. I held up my head and breathed cheerfully. All the cells in my body sensed such a beauty that I did not experience until that moment. I was delighted as I was nearer to the light, then suddenly I came back with a jerk.

As I opened my eyes I saw my worried sister beside me, and next to her was a nurse who tried to wake me up. I was still ecstatic about my short journey when I opened my eyes and I looked at the people with smile on my lips. Though after a while that feeling faded away, the beauty of the moment did not leave me.

I cannot rationalize what had happened to me; I experienced the flowing or a kind of slipping into an unknown and delightful realm. Nobody understands our experience but those who had sensed it before.

My world view about life has changed completely after my NDE. The afterlife was unknown to me, and this lack of knowledge scared me. I had a lot of questions in mind about death and the existence of the other world. But this experience left me with the great feeling about the living and death altogether. Now I really love this world with all its misery and calamity. I know that I am a passenger in this world and I must live a good life because we are not returned here anymore. So I try my best to have a lovely life, I love all the people respectfully, and I am waiting for a peaceful life on the other side.

Before my experience I was a religious person but my view about death was fearful and I felt everything will be annihilated through 
death. But this perspective is changed now; and I truly attempt to enjoy every moment and I try to enhance my soul and be prepared to leave here at any time.

\section{Discussion and Conclusion}

Our study had some limitations. First, because our sample size was small, we are not able to generalize the study results to all Shiite Muslims who survived a close brush with death. Translation is another problematic issue in this research. The Persian translation of the NDE Scale may not convey the full meaning of its English language questions to the Persian-speaking subjects in this study's survey.

Keeping these limitations in mind, we found from our investigation of Iranian Shiite Muslim NDEs that Muslim NDEs do occur and that they are not rare. In addition, we found that contents of our participants' NDEs contained the same features-OBE, passing through a tunnel, encountering an unearthly light-as contents of Western NDEs (Zingrone \& Alvarado, 2009). Our global impression of our participants' NDE narratives was that they seemed to us to be similar to Western NDErs' narratives; among the common features was ineffability-difficulty describing the experience in human language, whether English among Western NDErs or Persian among nonWestern ones. Similarly, like Western survivors of close brushes with death, our participant NDErs and CBrs did not differ from each other with regard to gender, age, education, or time since close brush with death (Holden, Long, \& MacLurg, 2009). Likewise, our NDEr participants described aftereffects reminiscent of those of Western NDErs (Noyes et al., 2009), including loss of fear of death, the paradox of looking forward to death while holding a conviction that life has purpose and a commitment to live life to its fullest, and a tendency to become more spiritual while becoming less affiliated with organized religion.

Results from previously published studies indicated a striking similarity in the contents of NDEs worldwide, including in non-Western countries (Long, 2010, pp. 160-170). Our results also suggest that because Shiite Muslim NDEs may not be especially different in their main elements from typical Western NDEs, they may not be heavily influenced by cultural or religious variations. Our findings therefore indicate that NDEs may be universal in their fundamental nature and, thus, may reflect transpersonal and transcendental phenomena rather than only materialistic and culturally based phenomena. If this finding is further substantiated by future investigations, the aware- 
ness that people from all cultures have similar experiences at the time of death could be an important tool for cross-cultural understanding and dialogue.

To our knowledge, ours is the first study to assess the possible NDE aftereffect of gratitude using a psychometrically sound instrument. Because our data were correlational in nature and assessed dispositional gratitude only post-NDE, we cannot say for sure that our NDEr participants were not more grateful even prior to their NDEs; in other words, we cannot claim that their NDEs resulted in their significantly greater dispositional gratitude. However, as many NDErs have described an increase in gratitude resulting from their NDEs (Noyes et al., 2009), we think it likely that our results reflect this dynamic. It remains for future researchers to investigate this dynamic more unequivocally, and based on our findings and both the psychometric quality and brevity of the GQ-6, we recommend this instrument for that purpose.

Future studies could focus on the content and aftereffects of NDEs using larger sample sizes of representative Muslim populations. Such research could enhance knowledge of Muslim NDEs and understanding of NDEs globally. NDEs might be an important spiritual concept that could help humanity toward more mutual understanding and global peace.

It is hoped that this study and future investigations of non-Western NDEs will advance understanding of NDEs. NDErs in non-Western cultures might face specific challenges openly expressing their NDEs as a result of the dearth of literature and public awareness. If so, studies of non-Western NDEs may advance appreciation of their challenges and hopefully help NDErs address such challenges to the greatest extent possible.

\section{References}

Cohen, J. (1988). Statistical power analysis for the behavioral sciences (2nd ed.). Hillsdale, NJ: Erlbaum.

Fracasso, C., Aleyasin, S., Friedman, H., \& Young, M. (2010). Near-death experiences among a sample of Iranian Muslims. Journal of Near-Death Studies, 29, 265-272.

Ghamarani, A., Kajbaf, M. B., Oreyzi, H. R., \& Amiri, S. (2008). The study of the validity and reliability of the Gratitude Questionnaire (GQ-6) in high school Students. Research in Psychological Health, 3(1), 77-86.

Greyson, B. (1983). The Near-Death Experience Scale: Construction, reliability, and validity. Journal of Nervous and Mental Disorders, 171(6), 369-375. 
Greyson, B. (1997). The near-death experience as a focus of clinical attention. Journal of Nervous and Mental Disease, 185, 327-334.

Greyson, B. (2001). Posttraumatic stress symptoms following near-death experiences. American Journal of Orthopsychiatry, 71, 368-373.

Greyson, B. (2003). Incidence and correlates of near-death experiences in a cardiac care unit. General Hospital Psychiatry, 25, 269-276.

Greyson, B. (2007). Consistency of near-death experience accounts over two decades: Are reports embellished over time? Resuscitation, 73, 407-411.

Holden J., Greyson, B., \& James, D. (2009). The field of near-death studies: Past, present, and future. In J. M. Holden, B. Greyson, \& D. James (Eds.), The handbook of near-death experiences: Thirty years of investigation (pp. 1-16). Westport, CT: Praeger/ABC-CLIO.

Holden, J., Long, J., \& MacLurg, J. (2009). Characteristics of Western near-death experiencers. In J. M. Holden, B. Greyson, \& D. James (Eds.), The handbook of near-death experiences: Thirty years of investigation (pp. 109-134). Westport, CT: Praeger/ABC-CLIO.

Kellehear, A. (2009). Census of non-Western near-death experiences to 2005: Observations and critical reflections. In J. M. Holden, B. Greyson, \& D. James (Eds.), The handbook of near-death experiences: Thirty years of investigation (pp. 135-158). Westport, CT: Praeger/ABC-CLIO.

Kreps, J. (2009). The search for Muslim near-death experiences. Journal of Near-Death Studies, 28, 67-86.

Long, J. (with Perry, P.). (2010). Evidence of the afterlife: The science of neardeath experiences. New York, NY: HarperCollins.

Loseu, S., Holden, J. M., Kinsey, L., \& Christian, R. (2014). The field of neardeath studies through 2011: An updated analysis of the scholarly periodical literature. Journal of Near-Death Studies, 31(4), 189-202.

McCullough, M., Emmons, R., \& Tsang, J. (2002). The grateful disposition: A conceptual and empirical topography. Journal of Personality and Social Psychology, 82, 112-127.

Nahm, M., \& Nicolay, J. (2010). Essential features of eight published Muslim near-death experiences: An addendum to Joel Ibrahim Kreps's "The search for Muslim near-death experiences". Journal of Near-Death Studies, 29, 255-264.

Nouri, F., \& Holden, J. (2008). Electromagnetic aftereffects of near-death experiences. Journal of Near-Death Studies, 27, 83-110.

Noyes, R., Fenwick, P., Holden, J. M., \& Christian, R. (2009). Aftereffects of pleasurable Western adult near-death experiences. In J. M. Holden, B. Greyson, \& D. James (Eds.), The handbook of near-death experiences: Thirty years of investigation (pp. 41-62). Santa Barbara, CA: Praeger/ABC-CLIO.

Zingrone, N. L., \& Alvarado, C. S. (2009). Pleasurable Western adult near-death experiences: Features, circumstances, and incidence. In J. M. Holden, B. Greyson, \& D. James (Eds.), The handbook of near-death experiences: Thirty years of investigation (pp. 17-40). Santa Barbara, CA: Praeger/ABC-CLIO. 